\title{
Recombinant growth hormone therapy in children with short stature in Kuwait: a cross-sectional study of use and treatment outcomes
}

Dalia Al-Abdulrazzaq ${ }^{1 *}$, Abdullah Al-Taiar ${ }^{2}$, Kholoud Hassan $^{3}$ and Iman Al-Basari ${ }^{3}$

\begin{abstract}
Background: Recombinant Growth hormone ( $\mathrm{rGH}$ ) therapy is approved in many countries for treatment of short stature in a number of childhood diagnoses. Despite the increasing body of international literature on rGH use, there is paucity of data on rGH use in Kuwait and the broader Middle-East which share unique ethnic and socio-cultural backgrounds. This study aimed to describe the pattern of use and treatment outcomes of rGH therapy in Kuwait.
\end{abstract}

Methods: This is a cross-sectional retrospective review of children treated with rGH in the Department of Pediatrics, in a major hospital in Kuwait between December 2013 and December 2014. Data were extracted using standard data extraction form and the response to rGH therapy was defined as a gain of $\geq 0.3$ standard deviation score (SDS) of height per year.

Results: A total of 60 children were treated with $\mathrm{rGH}$ in the center. Their Median (Interquartile) age at $\mathrm{rGH}$ initiation was $9.0(6.2,10.7)$ years. The most common indications for rGH therapy were Growth Hormone Deficiency (GHD) 23 (38.3 \%), Idiopathic Short Stature (ISS) 12 (20.0\%) and Small for Gestational Age (SGA) 9 (15.0\%). After excluding patients with TS, no significant differences were found in gender of those who received $\mathrm{rGH}$ therapy in all indications combined or in each group ( $p \geq 0.40$ ). At 1 -year follow-up, children in all groups had median height SDS change of $\geq 0.3$ SDS except for children with ISS. Age at $\mathrm{rGH}$ initiation was negatively associated with 1-year treatment response, Adjusted odds ratio (AOR) 0.56 (95 \% Cl: 0.04-1.49); $p=0.011$ ).

Conclusions: GHD is the most common indication of rGH therapy. All indications except for ISS showed significant 1-year treatment response to therapy. Treatment outcomes in patients with ISS should be further investigated in Kuwait. Younger age at initiation of rGH therapy was independently associated with significant response to therapy suggesting the importance of identifying children with short stature and prompt initiation of rGH therapy.

Keywords: Short Stature, Growth Hormone, Therapy, Kuwait, Middle East

\footnotetext{
* Correspondence: d.alabdulrazzaq@hsc.edu.kw

'Department of Pediatrics, Faculty of Medicine, Kuwait University, PO Box 24923, Safat 13110, Kuwait

Full list of author information is available at the end of the article
} 


\section{Background}

Recombinant Growth Hormone (rGH) has been used to promote linear growth since 1985. rGH acts mainly through increasing the secretion of insulin-like growth factor-1 (IGF-1) [1]. The indications for $\mathrm{rGH}$ have gradually been extended from replacement therapy in Growth Hormone Deficiency (GHD) to an increasing number of conditions in which short stature is not due to GHD. The new indications include Small for Gestational Age (SGA), Idiopathic Short Stature (ISS) and Turner Syndrome (TS) in addition to other indications [1-3]. Since rGH was introduced, there have been several observational studies that described its effectiveness among children [4-7]. In most of the indications listed above, rGH therapy has been shown to improve final adult height [5, 7-9]. Despite the increasing body of international literature on rGH use, there is paucity of data on rGH use in Kuwait and the broader Middle-East which share unique ethnic and socio-cultural backgrounds.

Although the use of rGH therapy was limited when it was gradually introduced in 2001, the use of rGH therapy has recently increased with the increasing number of trained pediatric endocrinologists in the country. The cost of treatment is covered by the government for Kuwaiti nationals and by a local Patient-Aid Organization for non-Kuwaiti nationals. In Kuwait, as in many other countries in the Gulf region, the pattern of use of $\mathrm{rGH}$ therapy and the treatment outcomes remain mostly unknown. It is possible that the treatment outcome differs between different ethnicities and extrapolation of data from other settings is not always appropriate. Furthermore, description of the pattern of use of rGH therapy and the treatment outcomes in Kuwait and comparing these to international practices and treatment outcomes, are critical for the healthcare system of Kuwait which will help improve quality of care and resource utilization. In this study, we aimed to describe the pattern of use of rGH in a pediatric endocrine center in Kuwait and evaluate the 1-year response to therapy. We aimed also to identify the factors associated with significant treatment response.

\section{Methods}

This is a cross-sectional retrospective review of children treated with $\mathrm{rGH}$ in the Department of Pediatrics, Mubarak Al-Kabeer Hospital, in Kuwait. These children were under follow-up at the Endocrine Clinic between December 2013 and December 2014. The Department of Pediatrics at this hospital is one of four departments which treat children with short stature. The department has a large coverage area in the country and serves a total pediatric population of approximately 250,000 [10]. The study was approved by the ethics committee of The
Health Sciences Centre, Kuwait University and the ethics committee at The Ministry of Health in Kuwait.

Children and adolescents treated with rGH were identified using pharmacy prescription records at the Hospital. They were categorized according to indication of therapy to five groups namely: Growth Hormone Deficiency (GHD), Idiopathic Short Stature (ISS), Small for Gestational Age (SGA), Turner Syndrome (TS) and variants, and other indications (e.g. syndromic short stature, chronic renal disease and inflammatory bowel disease). Growth hormone deficiency was diagnosed based on two stimulation tests with clonidine and glucagon. A peak GH concentration after the stimulation of $<20 \mathrm{mIU} / \mathrm{L}$ (approximately equivalent to $7.5 \mathrm{ng} / \mathrm{dL}$ ) was considered diagnostic of GHD. ISS was defined by a height below -2 SDS without any findings of underlying disease as evident by a complete evaluation by a pediatric endocrinologist including stimulated GH levels [11]. SGA was diagnosed when birth weight is less than the $10^{\text {th }}$ centile for gestational age [12]. rGH therapy was initiated at a dose of $25-30 \mu \mathrm{g} / \mathrm{kg} /$ day for children with GHD [2], $50 \mu \mathrm{g} / \mathrm{kg} /$ day for children with ISS [11], $35 \mu \mathrm{g} / \mathrm{kg} / \mathrm{day}$ for children with SGA [13], and $50 \mu \mathrm{g} / \mathrm{kg} /$ day for children with TS and chronic renal disease [2].

The medical records of identified patients were reviewed and data were extracted using standard data extraction form. The data included baseline information at $\mathrm{rGH}$ initiation such as demographic data, indication of $\mathrm{rGH}$ therapy, anthropometric measures (weight, height, and body mass index [BMI]) expressed as standard deviation scores (SDS) determined by the World Health Organization child growth standards that are approved for use in Kuwait by the Ministry of Health [14]. Data were also extracted on pubertal status of each patient which was documented according to the Tanner stage. Pre-pubertal status was defined as Tanner stage 1 breast development for girls and testicular volume of $<4 \mathrm{~mL}$ for boys. For patients with missing data on pubertal status in their medical records, girls younger than the age of 8 years and boys younger than the age of 9 years at initiation of rGH were considered pre-pubertal. Data at 1-year follow-up were also extracted from medical records. These include anthropometric measures as above; and a significant response to rGH therapy at follow-up was defined as a gain of $\geq 0.3$ SDS of height per year as per Ranke and Lindberg [15]. Pre-pubertal status at 1-year follow-up was defined as above; and missing data on pubertal status were also handled as above.

Statistical analysis was performed using STATA software13.1. Differences with p-value of less than 0.05 were deemed to be statistically significant. Continuous variables were expressed as mean (SD) when normally distributed or median (interquartile range: IQR) otherwise. Kruskal-Wallis test or ANOVA was used to test 
for the differences in continuous variables as appropriate; while Fisher's exact test was used to test for differences in categorical variables. We also tested the differences in the distribution of gender in each treatment group using exact binomial probability test. Multiple logistic regression was used to investigate the baseline factors associated with significant response to rGH therapy at 1-year follow-up.

\section{Results}

Sixty patients were treated with rGH during the study period of whom 31 (51.7\%) were males. After excluding patients with TS from the analysis, no significant difference was found in gender of those who received rGH therapy in all indications combined or in each group $(p \geq 0.40)$ although only 9 out of 23 patients with GHD were females $(p=0.40)$. Moreover, there was no significant difference in baseline height SDS between males and females (all indications $p=0.90$ ). Of the sixty patients, 44 (73.3\%) were Kuwaitis; and the most common indication for therapy was GHD (Fig. 1). The baseline characteristics of all the patients according to indication of therapy are summarized in Table 1. There were no significant differences between the treated groups in relation to age, nationality, pubertal status, height SDS, and BMI SDS at the initiation of rGH therapy.

A total of 44 patients had documented data on 1-year treatment response. Data on the characteristics of the patients and the parameters of the treatment response at 1 -year follow-up are shown in Table 2. For all indications combined, 33 patients $(75.0 \%)$ had significant response to $\mathrm{rGH}$ (i.e. height SDS change $\geq 0.3$ ). Children in all groups had a median height SDS change of $\geq 0.3$ SDS following the first year of therapy except for children with ISS who had a median height SDS change of $0.17(p=0.005)$. Moreover, only 4 out of 10 children (40\%) with ISS had a significant response to rGH

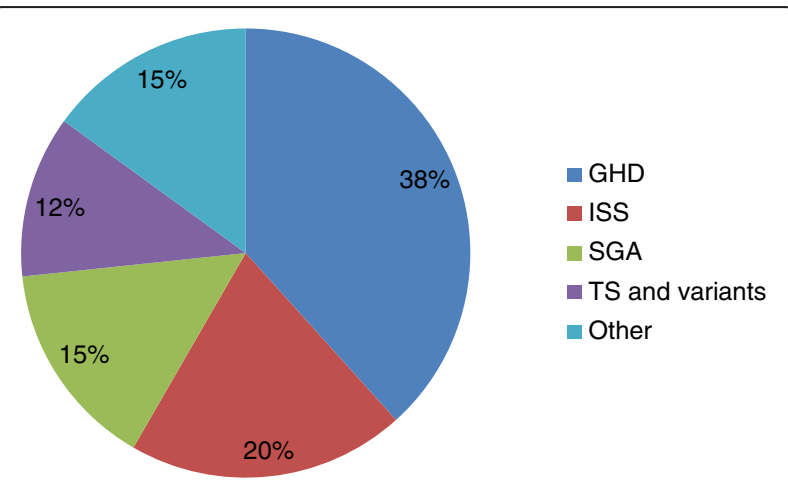

Fig. 1 Indications of rGH Therapy in Kuwait. rGH: Recombinant Growth Hormone ; GHD: Growth Hormone Deficiency; ISS: Idiopathic Short Stature; SGA: Small for Gestational Age; TS: Turner Syndrome compared to other indications $(p=0.012)$. The median height SDS change observed ranged from a minimum of 0.17 in children with ISS to a maximum of 0.74 in children with SGA. At 1-year follow-up, there were no significant differences in puberty status and BMI change between the groups. No adverse effects were reported in the medical records except for one patient who complained of headache. Patients with no documented 1-year follow-up data had baseline characteristics similar to the rest of the study group [median (IQR) age 9.6(6.2-11.2) year; 8 are females].

Table 3 shows the association between baseline factors and significant response to $\mathrm{rGH}$ therapy using logistic regression. Age at $\mathrm{rGH}$ initiation was negatively associated with significant 1-year treatment response, Adjusted odds ratio (AOR) 0.56 (95\% CI: 0.36- $0.87 ; p=0.011$ ). Sub-group analysis to investigate the association between baseline factors and significant response to $\mathrm{rGH}$ in each group could not be performed due to small numbers. When the analysis was restricted to GHD or ISS, there was no significant association between the age at initiation of rGH therapy and significant response to the treatment, AOR for age at initiation 0.07 (95\% CI: $0.34-1.43, p=0.326$ ) and 0.22 (95\% CI: $0.02-2.76, p=0.241)$, respectively.

\section{Discussion}

This study aimed to describe the pattern of use of rGH and the treatment outcomes. The study revealed that the most common indication for the use of $\mathrm{rGH}$ among children with short stature in Kuwait is GHD, which is similar to other reports from Europe and North America $[4,16]$. However, different from patients reported in the literature, our patients' population received rGH therapy at an earlier age for all indications (9.0 years) compared to the French SAGhE Study (11.0 years) [17]. Also, children with GHD in our study started rGH therapy at median age of 6.4 years compared to 10.2 years from the International ANSWER Registry [4], 10.8 years from USA [18], 9.2 years from the Pfizer International Growth Database (KIGS) [19] and 8.25 years from Taiwan [20]. For children with ISS, the age of initiation of therapy in a systematic review ranged from 6.1 to 12.9 years [21] compared to 10.6 years in our study. Another striking difference between our patients and patients described in the literature is the absence of gender difference in children receiving $\mathrm{rGH}$ in all indication groups combined or in each group separately. It has been reported elsewhere that males are more likely to receive $\mathrm{rGH}$ therapy compared to females $[22,23]$, which might be due to either parental gender preferences that affect seeking the treatment or differential referral. Equal numbers of males and females receiving rGH therapy in all indications suggest that parental gender preferences 
Table 1 Baseline characteristics at initiation of rGH Therapy

\begin{tabular}{|c|c|c|c|c|c|c|c|}
\hline Variable & Total $N=60$ & GHD $n=23$ & ISS $n=12$ & SGA $n=9$ & TS and variants $n=7$ & Others $n=9$ & $P$ value \\
\hline Female, n (\%) & $29(48.3 \%)$ & 9 (39.1\%) & $6(50.0 \%)$ & $4(44.4 \%)$ & $7(100.0 \%)$ & 3 (33.3 \%) & $0.917^{*}$ \\
\hline $\begin{array}{l}\text { Nationality, } \\
\text { Kuwaitis n (\%) }\end{array}$ & $44(73.3 \%)$ & $16(69.6 \%)$ & $7(58.3 \%)$ & $8(88.9 \%)$ & $4(57.1 \%)$ & 9 (100.0\%) & 0.122 \\
\hline $\begin{array}{l}\text { Age years, } \\
\text { median }(\mathrm{IQR})^{\star}\end{array}$ & $9.0(6.2,10.7)$ & $6.4(5.5,10.7)$ & $10.6(8.1,10.9)$ & $7.8(7.2,9.7)$ & $9.9(5.9,10.7)$ & $8.4(5.0,12.4)$ & 0.407 \\
\hline $\begin{array}{l}\text { Pre-pubertal, } \\
\text { n/N (\%) }\end{array}$ & $50 / 59(83.3 \%)$ & 21/23 (91.3 \%) & 10/12 (83.3 \%) & 9/9 (100.0 \%) & 5/7 (71.4\%) & $5 / 8(62.5 \%)$ & 0.127 \\
\hline $\begin{array}{l}\text { Height SDS, } \\
\text { mean (SD) }\end{array}$ & $-2.77(0.55)$ & $-2.76(0.50)$ & $-2.84(0.52)$ & $-2.49(0.54)$ & $-2.82(0.69)$ & $-3.03(0.69)$ & 0.432 \\
\hline $\begin{array}{l}\text { BMI SDS, } \\
\text { median (IQR) }\end{array}$ & $-0.46(-1.25,0.10)$ & $-0.32(-1.25,0.27)$ & $-0.44(-1.91,-0.04)$ & $-0.94(-2.48,-.58)$ & $0.10(-0.31,0.65)$ & $-0.27(-0.71,0.24)$ & 0.176 \\
\hline
\end{tabular}

rGH: Recombinant Growth Hormone ; GHD: Growth Hormone Deficiency; ISS: Idiopathic Short Stature; SGA: Small for Gestational Age; TS: Turner Syndrome. SD: Standard Deviation; SDS: Standard Deviation Score; BMI: Body Mass Index; *test was done excluding TS. "Missing for 1 case (other indications); "Missing for 5 cases ( 2 TS and 3 other indications); "Missing for 5 cases (2 TS and 3 other indications).

and differential referral are not strong in our setting. Nevertheless, it should be noted that in our study only 9 out of 23 patients with GHD were females. Although not statistically significant $(p \geq 0.40)$, influence of gender on referrals and seeking rGH therapy may warrant further investigation in our setting. Furthermore, gender differences in baseline height SDS were reported in the literature, where female subjects had lower mean height SDS compared to males across all indications groups which was not found in our patients' population [18]. This gender difference has been also attributed to referral bias as male children are usually recognized to have short stature earlier than female children [24].

In our study, children with GHD were shorter (SDS mean -2.76) compared to patients reported from Europe and North America (i.e. The International ANSWER Registry SDS mean -2.3 [4], and USA -2.2 [18]) but were of similar heights to patients from Taiwan (SDS mean -2.78) [20]. On the other hand, those with ISS were shorter (SDS mean -2.84) than those reported in the International ANSWER Registry (SDS mean -2.3) [4], the French SAGhE Study (SDS mean - 2.7) [17] and USA (SDS mean -2.3) [18]. However, children with SGA were taller (SDS mean -2.49) than children in other studies such as the International ANSWER Registry (SDS mean -3.1) [4], USA (SDS mean -2.6) [18], and Denmark (SDS median -3.0) [25]. It is not clear if such differences reflect genuine variation in patients' profile between our setting and other settings, and thus these differences should be interpreted cautiously because of the small numbers.

At 1-year follow-up, all groups had on average significant increment in height except for children with ISS with only 4 patients out of 10 having significant response to $\mathrm{rGH}$ therapy. It has been reported that the effectiveness of rGH for children with ISS is on average less than that achieved in other conditions for which the therapy is licensed [21]. This is why there is no unified consensus on the use of rGH for children with ISS. While the International Society of Pediatric Endocrinology and the Growth Hormone Research Society recommend rGH therapy for these children [11], the European Agency for Evaluation of Medicinal Products has not yet approved the use of rGH in children with ISS [9]. It has been suggested that the age at initiation of rGH therapy is negatively associated with the response to $\mathrm{rGH}$ therapy in various indications including ISS $[26,27]$. The Consensus Statement on Diagnosis and Treatment of Children with ISS indicates that the optimal age for initiation of treatment is five years to early puberty [11]. It should be noted that children with ISS started receiving $\mathrm{rGH}$ therapy at a relatively older age in our setting. The median age of this group was 10.6 years, which makes this group older than any other treatment group in our setting (Table 1), and also near the upper limit of the recommended age range for rGH therapy (5 years to early puberty) [11]. Results from the metaanalysis of 10 trials with 1-year follow-up data on response of children with ISS to rGH therapy showed a mean change of height SDS of 0.43 which is higher compared to the response of children in our study [28]. This may indicate a different unknown etiology of short stature in children with ISS in our region. ISS is diagnosed when children with short stature have no evidence of systematic, endocrine, nutritional, or chromosomal abnormalities [29]. Therefore, ISS is not a single homogenous disease entity with a well-known underlying cause. It may include normal variants of growth, such as familial short stature and constitutional delay of growth and puberty, both characterized by achievement of an adult height within the range of mid-parental height [21]. These variants in growth have not been studied in children in Kuwait and might be of different nature compared to other children in different parts of the world. Our data highlight the need for further investigation of the treatment outcome of $\mathrm{rGH}$ in children with ISS in our setting. 
Table 2 Characteristics of patients at 1-year follow-up with rGH Therapy

\begin{tabular}{|c|c|c|c|c|c|c|c|}
\hline Variable & Total $n=44$ & GHD $n=18$ & ISS $n=10$ & SGA $n=9$ & TS and variants $n=4$ & Others $n=3$ & $P$ value \\
\hline Female, $n(\%)$ & $21(47.7 \%)$ & 7 (39.0 \%) & $5(50.0 \%)$ & $4(44.4 \%)$ & $4(100.0 \%)$ & $1(33.3 \%)$ & $0.279^{*}$ \\
\hline Age in years, median (IQR) & $9.7(7.2,11.6)$ & $7.3(6.6,11.6)$ & $11.6(9.7,12.1)$ & $8.9(8.1,10.7)$ & $10.7(8.6,11.4)$ & $7.7(3.7,14.7)$ & 0.230 \\
\hline Pre-pubertal, n/N (\%) & 30/37 (81.1 \%) & $14 / 16(87.5 \%)$ & 6/9 (66.7 \%) & 6/7 (85.7 \%) & $2 / 3(66.7 \%)$ & $2 / 2(100.0 \%)$ & 0.641 \\
\hline Height SDS, mean (SD) & $-2.14(0.59)$ & $-2.11(0.57)$ & $-2.49(0.55)$ & $-1.82(0.59)$ & $-2.28(0.73)$ & $-1.98(0.21)$ & 0.158 \\
\hline Height SDS change, median (IQR) & $0.54(0.29,0.75)$ & $0.57(0.33,0.83)$ & $0.17(0.16, .41)$ & $0.74(0.59,0.76)$ & $0.40(0.22,0.61)$ & $0.77(0.44,0.93)$ & 0.005 \\
\hline BMI SDS, median (IQR) & $-0.73(-1.57,-0.21)$ & $-0.73(-1.34,-0.36)$ & $-0.51(-1.22,-0.09)$ & $-1.59(-2.00,-1.05)$ & $-0.31(-1.61,1.02)$ & $-0.19(-0.59,0.35)$ & 0.136 \\
\hline BMI change, median (IQR) & $-0.04(-0.49,0.24)$ & $-0.23(-0.49,0.07)$ & $0.19(-0.34,0.59)$ & $0.17(-0.59,0.48)$ & $-0.32(-1.01,0.43)$ & $-0.36(-0.43,1.06)$ & 0.431 \\
\hline Significant responders, n (\%) & $33(75.0 \%)$ & 15 (83.3\%) & $4(40.0 \%)$ & $9(100.0 \%)$ & $2(50.0 \%)$ & $3(100.0 \%)$ & 0.012 \\
\hline
\end{tabular}

rGH Recombinant Growth Hormone, GHD Growth Hormone Deficiency ISS Idiopathic Short Stature, SGA Small for Gestational Age, TS Turner Syndrome. SD Standard Deviation, SDS Standard Deviation Score, BMI Body Mass Index. *test was done excluding TS. 
Table 3 Association between baseline factors and significant response to $\mathrm{rGH}$ at 1-year follow-up in 44 patients

\begin{tabular}{llc}
\hline Baseline factors at rGH initiation & Odds Ratio $[95 \% \mathrm{Cl}]$ & $P$ value \\
\hline Male Gender & $0.24[0.04-1.49]$ & 0.127 \\
Age at initiation & $0.56[0.36-0.87]$ & 0.011 \\
Pre-pubertal at initiation of therapy & $0.06[0.001-1.98]$ & 0.114 \\
Height SDS at initiation & $2.61[0.41-16.80]$ & 0.310 \\
BMI SDS at initiation & $0.49[0.22-1.12]$ & 0.092 \\
\hline
\end{tabular}

$r G H$ Recombinant Growth Hormone, SDS Standard Deviation Score, BMI Body Mass Index; *Height SDS change $\geq 0.3$.

It has been shown that total gain in height SDS correlates significantly with pre-pubertal gain in height SDS and younger age at start of treatment in GHD [4, 18, 26]. Therefore, the Growth Hormone Research Society advises that treatment should be initiated as soon as the diagnosis is made although no specific optimal time was specified for children diagnosed with GHD [1, 2, 30]. Early age at start of therapy was identified as a predictor of adult height in children with ISS as well [18, 21, 26, 27]. In the present analysis, it was found- as reported in the literature- that younger age at initiation of therapy is significantly associated with significant response to the treatment in all indications combined. However, due to small sample size, such relation was not statistically significant in children with GHD and ISS. Furthermore, gender was not significantly related to the response to $\mathrm{rGH}$ in the present study, which is consistent with larger studies investigating response to rGH therapy such as the KIGS database [31]. Anthropometric measures at initiation of therapy have been suggested to predict positive response to $\mathrm{rGH}$; namely lower baseline height and higher BMI [32]. Such association was not demonstrated in our study, where baseline height and BMI were not significantly related to treatment response. This might reflects the different nature of growth in children in our region compared to other parts of the world.

Our study has several limitations including small number of patients and short period of follow-up. Because of this we were unable to detect meaningful differences. Our data also were obtained from one pediatric endocrine center, although a large center, but this might limit the generalizability of the results to other centers in the country and the region. The study was a cross-sectional retrospective review which relied on obtaining data from medical records. There was no data on several parameters (e.g. mid-parental height, target height, bone age, IGF-1 and IGF-BP3 levels, and $\mathrm{GH}$ doses at 1-year follow-up) which might influence response to $\mathrm{rGH}$ therapy. We have 16 patients with no follow-up data of whom eight have not reached 1-year of follow since the treatment. Their baseline characteristics are similar to the rest of the study group. Despite this, our study has several strengths. This study was conducted in one pediatric endocrine center which led to less variability in anthropometric measurements. Furthermore, it was an observational study of a real-life use of rGH rather than a controlled trial of therapy which cannot be ideally translated into everyday clinical practice.

\section{Conclusion}

In this analysis, we have demonstrated that GHD is the most common indication of rGH therapy; and that there is minimal gender difference in receiving $\mathrm{rGH}$ therapy in Kuwait. Our patients seem to receive rGH therapy at a younger age (except for ISS) compared to other settings. Height SDS change at 1-year of rGH therapy showed significant response in all indications except for patients with ISS. rGH treatment outcomes in patients with ISS should be further investigated in Kuwait. Younger age at initiation of rGH therapy was independently associated with significant response to the treatment at 1-year follow-up. This suggests the importance of identifying children with short stature and prompt rGH treatment which is more affordable in Kuwait than in other settings in the Middle East. Future multi-center longitudinal study with adult height data is needed to investigate the treatment outcomes of rGH therapy in children in Kuwait and the region. In order to identify factors predicting response to therapy, the study should include parameters such as mid-parental height, target height, bone age, IGF-1 and IGF-BP3 levels, GH doses, and genetic studies. This is critical to guide decision makers to optimally use the resources for healthcare.

\section{Abbreviations}

AOR: Adjusted odds ratio; BMl: Body mass index; GHD: Growth Hormone Deficiency; IGF-1: insulin-like growth factor-1; IGF-BP3: insulin -like growth factor - binding protein 3; ISS: Idiopathic short stature; rGH: Recombinant Growth Hormone; SDS: standard deviation score; SGA: small for gestational age; TS: Turner syndrome.

\section{Competing interests}

The authors declare no competing interests.

\section{Authors' contributions}

DA: Designed the study, contributed to data collection, analysis, interpretation, and drafted the manuscript. AA: Contributed to data analysis, interpretation and drafting of the manuscript. KS: Contributed to data collection and interpretation. IA: Contributed to study design, data collection, interpretation and drafting of the manuscript. All authors read and approved of the manuscript.

\section{Author's information}

DA is a certified Pediatric Endocrinologist who completed her paediatric residency at the Hospital for Sick children in Toronto, Ontario, Canada (RCPC). She then completed her pediatric training in Endocrinology and Metabolism at the Hospital for Sick Children in Toronto. Ontario, Canada (RCPC). She is currently an Assistant Professor at the Department of Pediatrics at the Faculty of Medicine, Kuwait University and a Pediatric Endocrinologist at the Department of Pediatrics at Mubarak Al-Kabeer Hospital, Kuwait. 


\section{Acknowledgments}

The authors gratefully acknowledge Professor. Adekunle Adekile, Department of Pediatrics, Faculty of Medicine, Kuwait University for his expert advice in drafting and preparing the manuscript. The authors also acknowledge Dr Fatemah Behbehani and the endocrine nurses at the Department of Pediatrics, Mubarak Al-Kabeer Hospital for their efforts in data collection.

\section{Author details}

${ }^{1}$ Department of Pediatrics, Faculty of Medicine, Kuwait University, PO Box 24923, Safat 13110, Kuwait. ${ }^{2}$ Department of Community Medicine, Faculty of Medicine, Kuwait University, PO Box 24923, Safat 13110, Kuwait. 'Department of Pediatrics, Mubarak Al-Kabeer Hospital, Ministry of Health, Safat, Kuwait.

Received: 21 July 2015 Accepted: 25 November 2015

\section{Published online: 03 December 2015}

\section{References}

1. Gharib H, Cook DM, Saenger PH, Bengtsson BA, Feld S, Nippoldt TB, et al. American Association of Clinical Endocrinologists medical guidelines for clinical practice for growth hormone use in adults and children-2003 update. Endocr Pract. 2003;9(1):64-76.

2. Wilson TA, Rose SR, Cohen P, Rogol AD, Backeljauw P, Brown R, et al. Update of guidelines for the use of growth hormone in children: the Lawson Wilkins Pediatric Endocrinology Society Drug and Therapeutics Committee. J Pediatr. 2003;143(4):415-21.

3. Richmond E, Rogol AD. Current indications for growth hormone therapy for children and adolescents. Endocr Dev. 2010;18:92-108.

4. Lee PA, Sävendahl L, Oliver I, Tauber M, Blankenstein O, Ross J, et al. Comparison of response to 2-years' growth hormone treatment in children with isolated growth hormone deficiency, born small for gestational age, idiopathic short stature, or multiple pituitary hormone deficiency: combined results from two large observational studies. Int J Pediatr Endocrinol. 2012; 2012(1):22.

5. Carel JC, Ecosse E, Nicolino M, Tauber M, Leger J, Cabrol S, et al. Adult height after long term treatment with recombinant growth hormone for idiopathic isolated growth hormone deficiency: observational follow up study of the French population based registry. BMJ. 2002;325(7355):70.

6. Hintz RL, Attie KM, Baptista J, Roche A. Effect of growth hormone treatment on adult height of children with idiopathic short stature. Genentech Collaborative Group. N Engl J Med. 1999;340(7):502-7.

7. Park HK, Lee HS, Ko JH, Hwang IT, Hwang JS. Response to three years of growth hormone therapy in girls with Turner syndrome. Ann Pediatr Endocrinol Metab. 2013;18(1):13-8.

8. Bannink E, Djurhuus CB, Christensen T, Jøns K, Hokken-Koelega A. Adult height and health-related quality of life after growth hormone therapy in small for gestational age subjects. J Med Econ. 2010;13(2):221-7.

9. Bryant J, Djurhuus CB, Christensen T, Jøns K, Hokken-Koelega A. Recombinant growth hormone for idiopathic short stature in children and adolescents. Cochrane Database Syst Rev. 2007;3, CD004440.

10. Public Authority for Civil Information (PACl), Population Statistical Reports. 2014 [cited 2015 July 12th]; Available from: https://www.paci.gov.kw/Home.aspx.

11. Cohen P, Rogol AD, Deal CL, Saenger P, Reiter EO, Ross JL, et al. Consensus statement on the diagnosis and treatment of children with idiopathic short stature: a summary of the Growth Hormone Research Society, the Lawson Wilkins Pediatric Endocrine Society, and the European Society for Paediatric Endocrinology Workshop. J Clin Endocrinol Metab. 2008;93(11):4210-7.

12. Lee PA, Chernausek SD, Hokken-Koelega AC, Czernichow P; International Small for Gestational Age Advisory Board. International Small for Gestational Age Advisory Board consensus development conference statement: management of short children born small for gestational age, April 24October 1, 2001. Pediatrics. 2003;111(6 Pt 1):1253-61.

13. Clayton PE, Cianfarani S, Czernichow P, Johannsson G, Rapaport R, Rogol A. Management of the child born small for gestational age through to adulthood: a consensus statement of the International Societies of Pediatric Endocrinology and the Growth Hormone Research Society. J Clin Endocrinol Metab. 2007;92(3):804-10.

14. Group, W.H.O.M.G.R.S. WHO Child Growth Standards based on length/height, weight and age. Acta Paediatr Suppl. 2006;450:76-85.

15. Ranke MB, Lindberg A, Board KI. Observed and predicted growth responses in prepubertal children with growth disorders: guidance of growth hormone treatment by empirical variables.

J Clin Endocrinol Metab. 2010;95(3):1229-37.

16. Bell J, Parker KL, Swinford RD, Hoffman AR, Maneatis T, Lippe B. Long-term safety of recombinant human growth hormone in children. J Clin Endocrinol Metab. 2010;95(1):167-77.

17. Carel JC, Ecosse E, Landier F, Meguellati-Hakkas D, Kaguelidou F, Rey G, et al. Long-term mortality after recombinant growth hormone treatment for isolated growth hormone deficiency or childhood short stature: preliminary report of the French SAGhE study. J Clin Endocrinol Metab. 2012;97(2):416-25.

18. Ross J, Lee PA, Gut R, Germak J. Factors influencing the one- and two-year growth response in children treated with growth hormone: analysis from an observational study. Int J Pediatr Endocrinol. 2010;2010:494656.

19. Darendeliler F, Lindberg A, Wilton P. Response to growth hormone treatment in isolated growth hormone deficiency versus multiple pituitary hormone deficiency. Horm Res Paediatr. 2011;76 Suppl 1:42-6.

20. Huang $Y H$, Wai $Y Y$, Van YH, Lo FS. Effect of growth hormone therapy on Taiwanese children with growth hormone deficiency. J Formos Med Assoc. 2012;111(7):355-63.

21. Deodati A, Cianfarani S. Impact of growth hormone therapy on adult height of children with idiopathic short stature: systematic review. BMJ. 2011;342:c7157.

22. Grimberg A, Huerta-Saenz L, Grundmeier R, Ramos MJ, Pati S, Cucchiara AJ, et al. Gender Bias in U.S. Pediatric Growth Hormone Treatment. Sci Rep. 2015;5:11099.

23. Grimberg A, Stewart E, Wajnrajch MP. Gender of pediatric recombinant human growth hormone recipients in the United States and globally. J Clin Endocrinol Metab. 2008:93(6):2050-6.

24. Hughes IP, Choong CS, Cotterill A, Harris M, Davies PS. Gender bias in children receiving growth hormone treatment. J Clin Endocrinol Metab. 2010;95(3):1191-8

25. Renes JS, Willemsen RH, Mulder JC, Bakker-van Waarde WM, Rotteveel J, Oostdijk W, et al. New insights into factors influencing adult height in short SGA children: Results of a large multicentre growth hormone trial. Clin Endocrinol (Oxf). 2015;82(6):854-61.

26. Reiter EO, Price DA, Wilton P, Albertsson-Wikland K, Ranke MB. Effect of growth hormone $(\mathrm{GH})$ treatment on the near-final height of 1258 patients with idiopathic GH deficiency: analysis of a large international database. J Clin Endocrinol Metab. 2006;91(6):2047-54.

27. Ranke MB, Lindberg A, Price DA, Darendeliler F, Albertsson-Wikland K, Wilton $P$, et al. Age at growth hormone therapy start and first-year responsiveness to growth hormone are major determinants of height outcome in idiopathic short stature. Horm Res. 2007;68(2):53-62.

28. Finkelstein BS, Imperiale TF, Speroff T, Marrero U, Radcliffe DJ, Cuttler L. Effect of growth hormone therapy on height in children with idiopathic short stature: a meta-analysis. Arch Pediatr Adolesc Med. 2002;156(3):230-40.

29. Ranke MB. Towards a consensus on the definition of idiopathic short stature. Horm Res. 1996;45 Suppl 2:64-6.

30. Growth Hormone Research S. Consensus guidelines for the diagnosis and treatment of growth hormone $(\mathrm{GH})$ deficiency in childhood and adolescence: summary statement of the GH Research Society. GH Research Society. J Clin Endocrinol Metab. 2000;85(11):3990-3.

31. Rose SR, Shulman DI, Larsson P, Wakley LR, Wills S, Bakker B. Gender does not influence prepubertal growth velocity during standard growth hormone therapy-analysis of United States KIGS data. J Pediatr Endocrinol Metab. 2005;18(11):1045-51.

32. Lee PA, Germak J, Gut R, Khutoryansky N, Ross J. Identification of factors associated with good response to growth hormone therapy in children with short stature: results from the ANSWER Program(R). Int J Pediatr Endocrinol. 2011;2011:6. 